\section{At the interfaces of spectroscopy}

\section{K.A. McLauchlan}

Computer Enhanced Spectroscopy: An

Editor-in-chief H.A. Willis.

Wiley-Heyden. 4/yr. $£ 65, \$ 130$.

DATA-handling by computer and computer interfacing to laboratory equipment are very much to the fore in the $1980 \mathrm{~s}$, and the basic idea of a new journal to cover such matters is sound. Computer Enhanced Spectroscopy (CES) deals with any use of a computer in any branch of spectroscopy. Its contents include data manipulation on- and off-line, library searching, design principles of systems and specific experimental devices; unusually, much software is reported. Authors are invited to publish details which went undescribed in an original scientific paper, a seductive call to double their number of publications.

The problems for the journal, though, are several. Such a range of specialist spectroscopic techniques is involved that the matter dealing with any one topic is dilute. Since in many research fields there exist dedicated journals, it seems unlikely
International Journal.

NEW JOURNALS

that a seminal paper in, for example, magnetic resonance will make its way to CES. For ideas of inter-disciplinary interest, on the other hand, there exist the well-respected Reviews of Scientific Instruments and Journal of Physics (E), which would take most of the material. Many of the practical accounts are deviceorientated and concern specific microcomputers and specific types of spectrometer. They are of limited use, for wherever such interfacing is necessary one finds that because of technical advances many problems must be solved afresh. Similarly, software is usually most efficiently written for the precise microcomputer and application in mind, and often not in a high-level language. Some of the basic data manipulation points made in the four issues of Vol. 1 are very straightforward.

The journal is well and attractively produced with excellent reproductions of a wide range of devices, flow diagrams and software. However the first volume does appear to have been short of material, which has perhaps led to the publication of some rather trivial articles. Because of this uncertain start, and the reservations expressed above, I cannot believe that it will become widely read.

K.A. McLauchlan is a Lecturer in the Physical Chemistry Laboratory, University of Oxford.

\section{Polymer exchange}

\section{Philip Hodge}

Reactive Polymers, Ion Exchangers, Sorbents.

Editor-in-chief F.G. Helfferich.

Elsevier Scientific. 8/yr. Dfl. 412, \$160.

FOR MOST of its history, polymer science has been mainly concerned with polymerization processes, the physical properties of polymers, and their applications in material science. There are many journals that cater for these areas. In contrast relatively little work has been carried out on synthetic reactive polymers. This is surprising, bearing in mind that nature makes extensive use of polymers in various recognition processes and to catalyse and control reactions between low molecular weight species.

In recent years interest in reactive polymers has increased considerably and Reactive Polymers, Ion Exchangers, Sorbents seeks to provide a forum for this

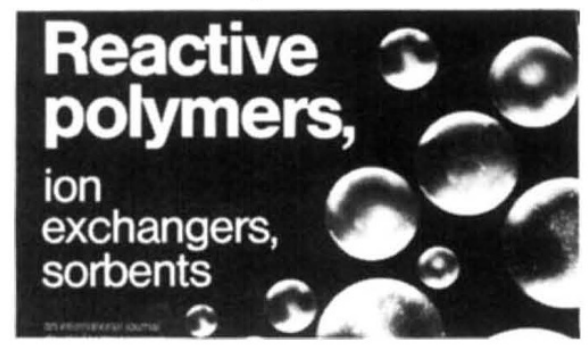

underdeveloped area of macromolecular science. The journal is well produced and contains original papers, short communications, reviews, book reviews, letters to the editor and a news page. For the first year it appeared quarterly but eight issues will appear in 1984, surely a sign of good health.

The standard of papers is excellent and there are contributions from many internationally known authors of good reputation. Thus far a rather high proportion of the papers has dealt with ion exchange resins, metal extraction using various sorbents and closely related topics. This bias probably reflects the fact that ion exchange resins are one of the few types of reactive polymers which have been studied for many years and have industrial applications. There are, however, many other areas of interest - polymer-supported catalysts, chemical modifications of synthetic and natural polymers, and biomedical and pharmaceutical applications, for example - and it is to be hoped that the number of papers dealing with these areas will increase to give the journal a better balance.

It is still hard to say how well this journal will fare in the long term. It serves a growing area of polymer science and if it can attract sufficient papers and publish them more frequently, whilst maintaining its present quality, it could do well.

Philip Hodge is a Reader in Organic Chemistry at the University of Lancaster.

\section{Separate ways}

Alwyn $\mathrm{M}^{\mathrm{c}} \mathrm{Kay}$

Solvent Extraction and Ion Exchange.

Editors E. Philip Horwitz and James D.

Navratil.

Dekker. 6/yr. $\$ 150$ (US), $\$ 166.50$

(elsewhere).

THE launching of Solvent Extraction and Ion Exchange (SEIE) may well be considered overdue, in view of the enormous expansion in this area since 1945. The journal is a companion to Separation Science and Technology, and its appearance follows a questionnaire addressed to specialists in the two related fields.

The first volume of four numbers, published during 1983, contains important material. There are 32 papers, five "notes" (not obviously distinguishable from papers), two valuable review articles and two letters; a bibliography and other items are included in each issue. Volume 2 (1984) will be larger, with eight issues, and it is expected that the journal will be expanded yet further. Certainly, there is plenty of scope for this.

There is, however, a long way to go before the editors' hope of the journal becoming the principal vehicle for papers on solvent extraction and ion exchange can be realized. At present SEIE carries only a small proportion of the total of papers on the subject being published, as the number of entries in the bibliography demonstrates. Moreover the selection is unbalanced in various ways. Academic chemical topics predominate, with emphasis on matters of nuclear interest, and there is little on equipment or industrial processes. On the other hand, the ratio of four solvent extraction papers to one ion exchange paper seems a fair one.

The journal is reproduced directly from the authors' typescripts and diagrams, but despite this it is not particularly cheap. The quality of presentation varies from very good to just adequate; in the worst cases one wishes that the method of printing did not preclude sub-editing to get rid of misprints, poor English and other blemishes. On the positive side, photographic reproduction should speed up publication, and must papers do appear reasonably promptly. The bibliography, however, being based on Chemical Abstracts rather than current journal contents lists, is unfortunately somewhat out-of-date.

$M y$ verdict is that SEIE indeed fills a gap. But there is some sacrifice of quality to economy and speed of production, and the editors would do well to keep an eye on their standards.

Alwyn McKay was formerly a Group Leader in the Chemical Technology Division of the Atomic Energy Research Establishment, Harwell, Oxfordshire. 